Check for updates

Cite this: RSC Adv., 2017, 7, 45029

Received 11th August 2017

Accepted 12th September 2017

DOI: 10.1039/c7ra08885j

rsc.li/rsc-advances

\section{A bifunctional luminescent europium-organic framework for highly selective sensing of nitrobenzene and 4-aminophenol $\dagger$}

\author{
Ming-Liang Gao, ${ }^{a}$ Xiao-Man Cao, ${ }^{a}$ Yu-Yang Zhang, ${ }^{a}$ Mei-Hong Qi, \\ Shi-Ming Wang, (D) ${ }^{b}$ Lin Liu $^{\star a}$ and Zheng-Bo Han (D) *a
}

A microporous hexanuclear europium cluster based MOF, $(\mathrm{DMA})_{2}\left[\mathrm{Eu}_{6}\left(\mu_{3}-\mathrm{OH}\right)_{8}(\mathrm{BPDC})_{6}\left(\mathrm{H}_{2} \mathrm{O}\right)_{6}\right]_{n} \cdot(\mathrm{solv})_{x}$ (DMA $=$ dimethylamine cation and $\mathrm{H}_{2} \mathrm{BPDC}=\left[1,1^{\prime}\right.$-biphenyl $]-4,4^{\prime}$-dicarboxylic acid), can be applied as a highly selective and sensitive bifunctional luminescence sensor to detect nitrobenzene and 4-aminophenol through an energy competition mechanism. More interestingly, this MOF can detect nitrobenzene and 4-aminophenol with low detection limits of 5-70 ppm and 5-110 ppm, respectively
With the rapidly growing population and industrialization, environmental pollution has become increasingly concerning throughout the world. In particular, organic pollutants have given rise to a series of overwhelming problems for nature. Therefore, efficient detection or removal of these organic pollutants has always been an active study area of environmental protection. Nitrobenzene (NB) and 4-aminophenol (4-AP) are distinctly important organic synthetic intermediates. ${ }^{1}$ In particular, NB is a common organic chemical in industrial manufacture. They are both irreplaceable for synthesizing multifarious chemical products, including azo dyes, medicines, agrochemicals, petroleum additives and epoxy curing agents. ${ }^{2}$ Moreover, they are representative potential environmental contaminants and possess high toxicity, which can cause dyspnea, skin eczema and dermatitis. ${ }^{3}$ Therefore, various detection methods, such as gas chromatography (GC), high performance liquid chromatography (HPLC) and ion mobility spectrometry are being applied for detecting and selective sensing of NB and 4-AP, etc. ${ }^{4}$ Due to their similar structural complexity and charge density etc., direct determination, especially selective detection of NB or 4-AP from their geometric isomers is an unmanageable task. ${ }^{5}$ Therefore, how to develop highly selectivity, specific sensing, quick response and easy readout of the analytical method is still hot research at present. Recently, luminescence sensor technology has been attracted more and more attention by researchers, which is owing to its high sensitivity and selectivity. ${ }^{6}$

${ }^{a}$ College of Chemistry, Liaoning University, Shenyang 110036, P. R. China. E-mail: ceshzb@lnu.edu.cn; liulin@lnu.edu.cn

${ }^{b}$ College of Light Industry, Liaoning University, Shenyang 110036, People's Republic of China

$\dagger$ Electronic supplementary information (ESI) available: Experimental details, PXRD, luminescence spectra and UV-vis spectra. See DOI: 10.1039/c7ra08885j
Metal-organic frameworks (MOFs) have been utilized as potent materials for gas adsorption, catalysis and sensing in recent years. ${ }^{1 b, 1 e, 7}$ Luminescent MOFs are a category of rapidly growing porous materials with an extensive range of applications. Particularly, rare-earth (RE) or RE cluster MOFs have attracted widespread interest, because of their characteristic luminescence property, artistic structure, topological diversity and well-organized channels. ${ }^{8}$ Therefore, an increasing number of RE-MOFs have been synthesized to be utilized in luminescence sensor area. ${ }^{9}$ Zhou et al. reported a robust microporous Eu-MOF, $\mathrm{Eu}_{3}(\mathrm{MFDA})_{4}\left(\mathrm{NO}_{3}\right)(\mathrm{DMF})_{3}$ $\left(\mathrm{H}_{2}\right.$-MFDA $=$ 9,9-dimethyl-fluorene-2,7-dicarboxylic acid $)$ for selective sensing of nitro explosives. ${ }^{10}$ In our previous work, $\left[\mathrm{Tb}(\mathrm{mtpc})_{1.5}(\mathrm{DMA})\left(\mathrm{H}_{2} \mathrm{O}\right)\right] \cdot 2 \mathrm{H}_{2} \mathrm{O} \quad\left(\mathrm{H}_{2} \mathrm{mtpc}=2^{\prime}, 4^{\prime}\right.$-dimethyl$1,1^{\prime}: 3^{\prime}, 1^{\prime \prime}$-terphenyl-4,4"-dicarboxylic acid) was synthesized and possessed excellent sensing ability for NB. ${ }^{11}$ In addition, gea-MOF1 was used as luminescent probe for sensing $\mathrm{NB}$ and aniline. ${ }^{12}$

Recently, a series of RE-MOFs, (DMA $)_{2}\left[\mathrm{RE}_{6}\left(\mu_{3}-\mathrm{OH}\right)_{8}-\right.$ $\left.(\text { BPDC })_{6}\right] \cdot x$ (solvent) $($ DMA $=$ dimethyl amine cation and BPDC $=\left[1,1^{\prime}\right.$-biphenyl $]-4,4^{\prime}$-dicarboxylic acid) were reported by us and these RE-MOFs exhibit excellent chemical stability in common organic solvents and were applied as luminescent barcode materials. ${ }^{13}$ In this work, $(\mathrm{DMA})_{2}\left[\mathrm{Eu}_{6}\left(\mu_{3}-\mathrm{OH}\right)_{8}(\mathrm{BPDC})_{6}\right]$. $x$ (solvent) (Eu-MOF) was used as luminescent sensor for efficient sensing of NB and 4-AP, which verified the outstanding potential of Eu-MOF as optical material.

Eu-MOF crystallizes in cubic space group $F m \overline{3} m$ and hexanuclear carboxylate cluster as a 12-connected secondary building block is linked by linear BPDC ligand to form a 3D microporous MOF. This Eu-MOF features two types of microporous cages, tetrahedral cages and octahedral cages, with the diameters estimated to be $1.2 \mathrm{~nm}$ and $1.6 \mathrm{~nm}$, respectively (Fig. 1). The crystalline structure of Eu-MOF was confirmed by PXRD patterns (Fig. S1, ESI $\dagger$ ). Thermogravimetric analysis was showed in Fig. S2, ESI. $\dagger$ 


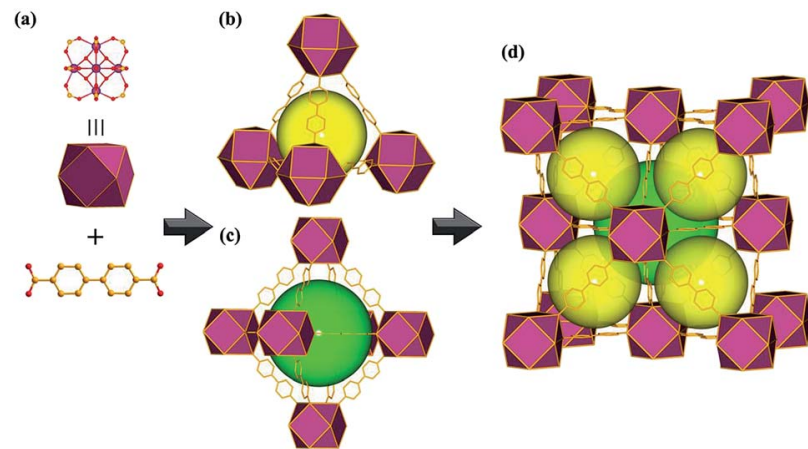

Fig. 1 (a) Ball and stick representation of the BPDC ligand and hexanuclear unit: $\left[\mathrm{Eu}_{6}\left(\mu_{3}-\mathrm{OH}\right)_{8}(\mathrm{COO})_{12}\right.$ ] (yellow: $\mathrm{C}$; red: O; violet: $\left.\mathrm{Eu}\right) ;(\mathrm{b})$ the terahedral cage; (c) the octahedral cage; (d) packing of two types of cages.

Eu-MOF contains conjugated $\pi$ moieties and $\mathrm{Eu}^{3+}$ ions. When the ultraviolet light irradiates, the conjugated $\pi$ moieties absorb ultraviolet light, simultaneously, efficiently transfer energy to $\mathrm{Eu}$ centres and sensitize $\mathrm{Eu}$ ions luminescence. Therefore, Eu-MOF is hopeful candidate for potential luminescence material, which aroused our interest to study its potential optics properties in this field. ${ }^{14}$ Solid state excitation and emission spectra of $\mathrm{H}_{2} \mathrm{BPDC}$ and Eu-MOF at room temperature are shown in Fig. S3 and S4, ESI. $\dagger$ When excited at $317 \mathrm{~nm}$, Eu-MOF display five strong characteristic luminescence emissions at 578, 592, 613,650, and $698 \mathrm{~nm}$ at room temperature which are ascribed to ${ }^{5} \mathrm{D}_{0} \rightarrow{ }^{7} \mathrm{~F}_{J}(J=0-4)$ transitions, respectively. The strongest character emission at $613 \mathrm{~nm}$ $\left({ }^{5} \mathrm{D}_{0} \rightarrow{ }^{7} \mathrm{~F}_{2}\right.$ transition $)$ is on account of electric dipole transition, which is called ultrasensitive transition and come into being red emission. Due to the diameters of cavities of Eu-MOF, small organic compounds can enter into the cavities of Eu-MOF, it is better for us to investigate the luminescence properties of this MOF. Therefore, the applications of sensing small organic compounds were investigated. For investigating the luminescence properties of Eu-MOF, the power samples were stable suspended in methanol (MeOH), ethanol (EtOH), acetone (AT), $\mathrm{N}, \mathrm{N}$-dimethylethylamine (DMF), $\quad \mathrm{N}, \mathrm{N}$-dimethylacetamide (DMA), acetonitrile (MeCN), $n$-hexane ( $n$-HX), tetrahydrofuran (THF), through treating by ultrasonication, respectively. Then, the luminescent measurements of suspension of Eu-MOFsolvents were proceeding, the result revealed that luminescence intensity of Eu-MOF-MeOH suspension was evident stronger than other organic solvents (Fig. S5, ESI $\dagger$ ). Therefore, the following experiments of luminescent sensing were utilized EuMOF-MeOH suspension.

Meanwhile, the detection experiments of selective luminescence sensing of aromatic compounds were carried out. Some aromatic compounds, such as toluene (TO), phenol ( $\mathrm{PhOH}$ ), chlorobenzene ( $\mathrm{Cl}-\mathrm{BZ})$, bromobenzene (Br-BZ), iodobenzene (I-BZ), $o$-xylene $(o$-XL), $p$-xylene $(p$-XL), $m$-xylene ( $m$-XL), ethylbenzene (E-BZ), antraceno (AN), NB, benzene (BZ), phenylcarbinol (PC), benzaldehyde (BD), acetophenone (AP), hydroquinone (HQ), $p$-bis(bromomethyl)benzene ( $p$-BB), styrene (SR) and naphthalene (NT) were added to the $\mathrm{MeOH}$ suspension of
Eu-MOF leading to the whole solution with same concentration (70 ppm), respectively (Fig. 2). $I_{0}$ is the original luminescence intensity and $I$ is the luminescence intensity in the presence of the analyte. The results indicate that only NB exhibites the most significant quenching effect on the luminescence intensities of MeOH suspensions of Eu-MOF. Thus, Eu-MOF could be applied to detect NB as a luminescence sensor.

In order to study the detection range of $\mathrm{NB}$, the detection limit of NB is imperative. It is further found that the emissive responses are gradually diminuted with increasing the amounts of NB (Fig. 3). This phenomenon implies that the luminescence quenching of NB possessed concentration-dependent. The luminescence intensity decreases $15.6 \%$ at $5 \mathrm{ppm}$ and almost completely quenching is detected at $70 \mathrm{ppm}$. The Stern-Volmer (S-V) equation can be used to explain the quenching efficiency: ${ }^{1 g} I_{0} / I=K_{\mathrm{SV}}[Q]+1,[Q]$ is the concentration of the analyte, and $K_{\mathrm{SV}}$ is the quenching constant $\left(\mathrm{ppm}^{-1}\right)$. At low concentration, the curve present good linear and subsequently deviate from linearity. At higher concentrations, the curve bend upwards, this phenomenon may attribute to the presence of simultaneous dynamic and static quenching. ${ }^{1 d}$ The $K_{\mathrm{SV}}$ was calculated as 0.044 (Fig. S6, ESI $\dagger$ ). These results reveal that EuMOF exhibites significant sensitivity and selectivity toward NB. To comparison with other MOFs sensors for NB, Eu-MOF possesses higher quenching efficiency (Table S1, ESI $\dagger$ ). Because of the luminescence response correlated with the $-\mathrm{NO}_{2}$ group, the luminescence quenching of Eu-MOF to various nitro compounds were investigated. 2,4-Dinitrophenol (DNP), $p$-nitrophenol ( $p$-NP), 4-nitrotoluene (4-NP), o-nitrophenol $(o$-NP), 3,5-dibromonitro benzene $(3,5-\mathrm{DB})$ and $p$-nitrobenzaldehyde ( $p$-ND) were selected to investigate the luminescence response of Eu-MOF (Fig. S7, ESI $\dagger$ ). The various nitro compounds with the same concentration $(70 \mathrm{ppm})$ were added into Eu-MOF-MeOH suspension, respectively and the quenching efficiencies of the nitro compounds are $92.3 \%(\mathrm{NB})>65.4 \%$ $(p-\mathrm{NP})>56.3 \%(4-\mathrm{NP})>46.6 \%(\mathrm{DNP})>44.1 \%(o-\mathrm{NP})>32.0 \%$ $(p-\mathrm{ND})>31.8 \%(3,5-\mathrm{BD})$. The results of the sensing tests clearly

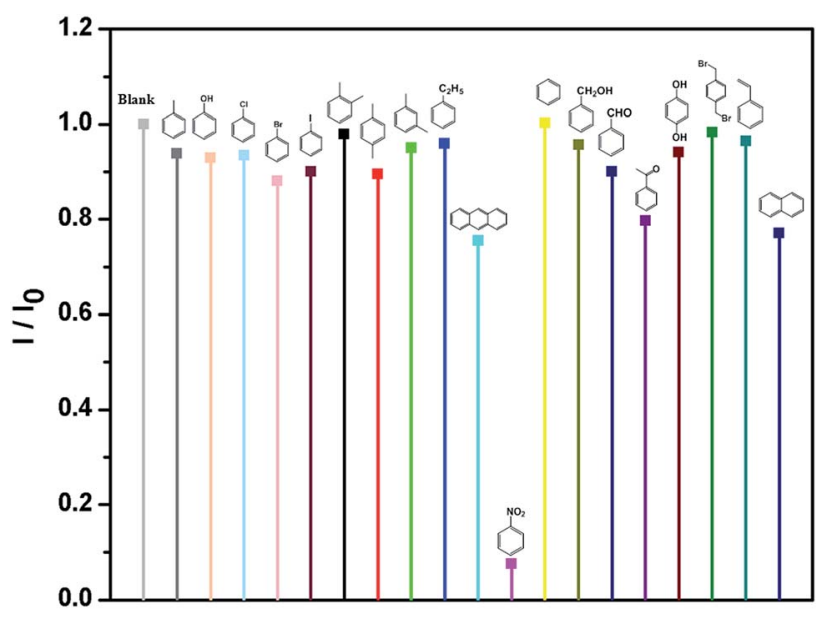

Fig. 2 Luminescent intensities of Eu-MOF at $613 \mathrm{~nm}$ in $\mathrm{MeOH}$ suspension of Eu-MOF with 70 ppm of different aromatic compounds $\left(\lambda_{\mathrm{ex}}=317 \mathrm{~nm}\right)$. 


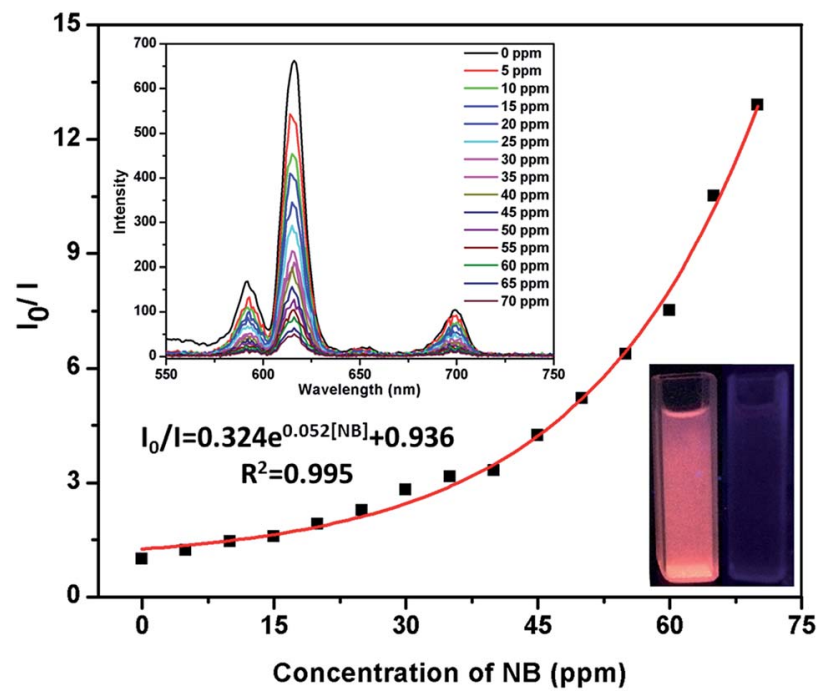

Fig. 3 Emission spectra of $\mathrm{MeOH}$ suspension of Eu-MOF at room temperature in the presence of 5-70 ppm of NB with respect to Eu-MOF $\left(\lambda_{\text {ex }}=317 \mathrm{~nm}\right.$ ). Inset: photograph showing the change of the original luminescence of $\mathrm{MeOH}$ suspension of Eu-MOF under ultraviolet light irradiation at $365 \mathrm{~nm}$ (the bottom right corner) and the concentration-dependent upon the addition of $\mathrm{NB}$ in the $\mathrm{MeOH}$ suspension of Eu-MOF (the top left corner).

verify the highest luminescence quenching efficiency by NB compares to other nitro compounds. As an excellent luminescence sensor, anti-interference, reproducible ability and structure stability tests of the material are necessary. ${ }^{15}$ Therefore, the selectivity of NB with various aromatic compounds presented were investigated. The experimental results show that Eu-MOF has high-efficiently sensing and selectivity towards NB (Fig. S8, ESI $\dagger$ ). Additionally, the cyclic tests of Eu-MOF to sense NB were studied. After 10 cycle tests, the quenching efficiency of Eu-MOF towards NB could still keep about 90\% (Fig. S9, ESI $\dagger$ ). Luminescence response results suggest that Eu-MOF possessed preeminent anti-interference ability and recyclability. Structure stability was confirmed by PXRD patterns, the result reveals that framework structure is maintained after 10 cycles (Fig. S10, $\mathrm{ESI} \dagger$ ).

As introduced above, Eu-MOF could selective and sensitive sense of NB and not sensitive to other aromatic or nitro compounds. To better understand the mechanism of luminescence quenching effect of NB, UV-vis absorb spectra of nitro compounds were investigated (Fig. S11, ESI $\dagger$ ). Spectral analysis implies that ultraviolet absorption band of NB partially overlaps with the excitation wavelength of Eu-MOF. To compare with other nitro compounds, there are no obvious ultraviolet absorption bands overlapping. Therefore, the mechanism of luminescence quenching could be attributed to energy competition mechanism between Eu-MOF and NB. The ultraviolet absorption bands of NB could reduce the excitation energy of Eu-MOF, consequently resulting in luminescence quenching. ${ }^{\mathbf{1 6}}$

4-AP is distinctly important organic synthetic intermediate for synthesizing multifarious chemical products, including paracetamol, clofibrate, sulfur dyestuff, stabilizer, etc. But 4-AP is also toxicant with double toxicity of aniline and phenol. It can be absorbed by the skin to cause dermatitis, methemoglobinemia and asthma etc. $^{1 e}$ Therefore, the detection of 4-AP in environment is crucial. In this investigation, ammonium hydroxide (AH), ethylenediamine (EDA), triethylamine (TEA), $N$-chlorosuccinimide $(\mathrm{N}-\mathrm{CN}), \quad N, N^{\prime}$-methylenebisacrylamide (MB), lsonicotinamide (LA), 4-aminobutyric acid (4-AA), cyanoguanidine (CA), succinimide (SM), methenamine (MA), o-phenylenediamine (o-PA), aniline (AN) and 4-AP were selected to test the luminescence sensor performance. The various amines were added into the MeOH suspension of Eu-MOF with the concentration of $110 \mathrm{ppm}$, respectively. It is interesting that luminescence quenching phenomenon is found by adding 4-AP (Fig. 4). The results imply that 4-AP could be detected and selective sensing by Eu-MOF through obvious luminescence response. In order to best study the sensing function towards 4-AP, the concentration dependent experiments of 4-AP were tested. The different concentration (5-110 ppm) of 4-AP were added into the MeOH suspension of Eu-MOF. As shown in Fig. 5, the luminescence intensity decreases $8.4 \%$ at $5 \mathrm{ppm}$ and almost completely quenching is detected at $110 \mathrm{ppm}$. The phenomenon of luminescence quenching is similar to NB. The $K_{\mathrm{SV}}$ was calculated as 0.026 (Fig. S12, ESI $\dagger$ ). There is a satisfactory non-linear relation between concentration and $I_{0} / I$. The anti-interference, reproducible ability and structure stability tests of Eu-MOF were also investigated. The anti-interference experimental results reveal that Eu-MOF shows outstanding anti-interference ability (Fig. S13, ESI†). The 10 cycles of recyclability experiments exhibit high recyclability for sensing of 4-AP (Fig. S14, ESI $\dagger$ ). The structure stability was also confirmed by PXRD patterns, after 10 cycles of recyclability experiments, the framework was maintained (Fig. S10, ESI $\dagger$ ). Therefore, Eu-MOF could be applied for sensing in the systems with complicated components with highly sensitive to 4-AP from other amines. In order to best understand the mechanism of quenching effect, the UV-vis spectrum of 4-AP was measured (Fig. S11, ESI $\dagger$ ), the absorption band of 4-AP is almost overlapped by the wide excitation bands of Eu-MOF. Therefore,

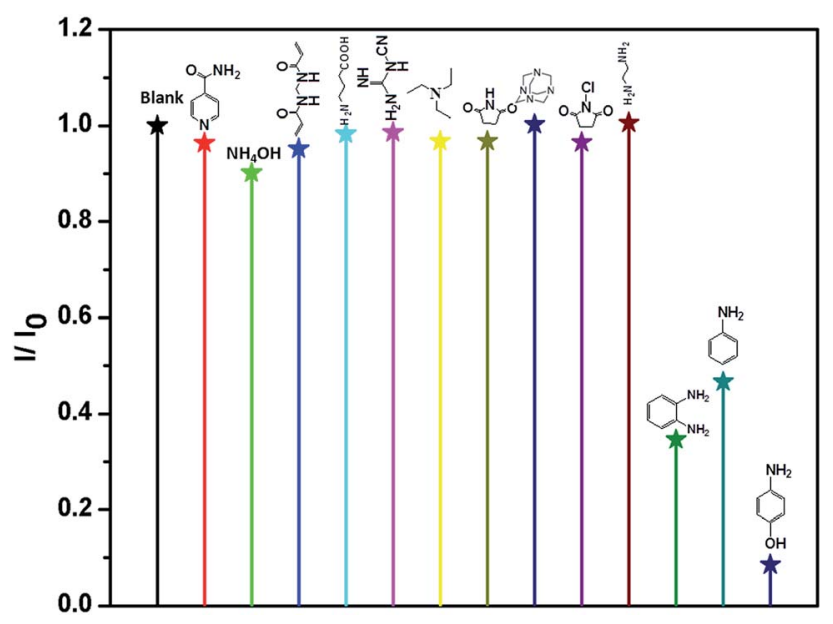

Fig. 4 Room-temperature luminescent intensity of Eu-MOF at $613 \mathrm{~nm}$ in $\mathrm{MeOH}$ suspension of Eu-MOF with 110 ppm of different organic amines $\left(\lambda_{\mathrm{ex}}=317 \mathrm{~nm}\right)$. 


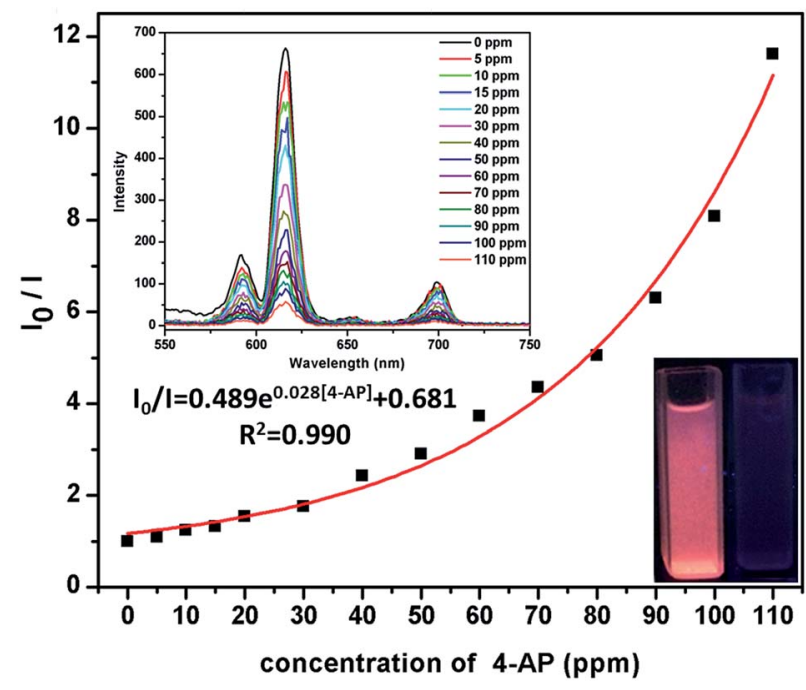

Fig. 5 Emission spectra of $\mathrm{MeOH}$ suspension of Eu-MOF at room temperature in the presence of 5-110 ppm of 4-AP with respect to Eu-MOF $\left(\lambda_{\text {ex }}=317 \mathrm{~nm}\right.$ ). Inset: photograph showing the change of the original luminescence of $\mathrm{MeOH}$ suspension of Eu-MOF under ultraviolet light irradiation at $365 \mathrm{~nm}$ (right) and the concentrationdependent upon the addition of 4-AP in the $\mathrm{MeOH}$ suspension of Eu-MOF (left).

mechanism of quenching effect might be attributed to energy competition mechanism between 4-AP and Eu-MOF. The UV-vis absorption of 4-AP could reduce the absorption of excitation energy of Eu-MOF at about $317 \mathrm{~nm} .{ }^{17}$

In summary, Eu-MOF exhibits potential capacity for selective sensing of NB and 4-AP with lower detection level, high recyclability and prominent anti-interference ability. Because of the neat topology and large channel within the cluster based Eu-MOF, NB and 4-AP might come into the cages of the MOF. It is beneficial to energy competition between analytes and Eu-MOF in biological and environmental analysis field. Therefore, our ongoing work will be concentrate on construct RE cluster MOF to apply to host-guest chemistry.

\section{Conflicts of interest}

The authors declare no competing financial interest.

\section{Acknowledgements}

This work was granted financial support from National Natural Science Foundation of China (Grant 21671090 and 21271096).

\section{Notes and references}

1 (a) J. Ghasemi and A. Niazi, Talanta, 2005, 65, 1168; (b) J. Ghasemi and A. Niazi, Talanta, 2005, 65, 1168; (c) W. P. Lustig, S. Mukherjee, N. D. Rudd, A. V. Desai, J. Li and S. K. Ghosh, Chem. Soc. Rev., 2017, 46, 3242; (d) F. Zhang, Y. Wang, T. Chu, Z. Wang, W. Li and Y. Yang, Analyst, 2016, 141, 4502; (e) J. Li, C. Y. Liu and Y. Liu, J. Mater. Chem., 2012, 22, 8426; $(f)$ Z. Hu, B. J. Deibert and
J. Li, Chem. Soc. Rev., 2014, 43, 5815; (g) S. W. Thomas, G. D. Joly and T. M. Swager, Chem. Rev., 2007, 107, 1339; (h) H. Reis, M. G. Papadopoulos and A. Grzybowski, J. Phys. Chem. B, 2006, 110, 18537.

2 K. M. Wollin and H. H. Dieter, Arch. Environ. Contam. Toxicol., 2005, 49, 18.

3 A. Konarev and V. Novikov, Russ. J. Electrochem., 2013, 49, 81. 4 (a) P. Vineis and R. Pirastu, Canc. Causes Contr., 1997, 8, 346;

(b) R. Benigni and L. Passerini, Mutat. Res., Rev. Mutat. Res., 2002, 511, 191.

5 (a) C. Tong, Y. Guo and W. Liu, Chemosphere, 2010, 81, 430; (b) A. S. Yazdi, F. Mofazzeli and Z. Eshaghi, J. Chromatogr. A, 2009, 1216, 5086; (c) Z. H. Xiang, C. Q. Fang, S. H. Leng and D. P. Cao, J. Mater. Chem. A, 2014, 2, 7662.

6 (a) H. Xu, F. Liu, Y. Cui, B. Chen and G. Qian, Chem. Commun., 2011, 47, 3153; (b) M. Guo and Z. M. Sun, J. Mater. Chem., 2012, 22, 15939; (c) S. S. Nagarkar, B. Joarder, A. K. Chaudhari, S. Mukherjee and S. K. Ghosh, Angew. Chem., Int. Ed., 2013, 52, 2881; (d) X. Zhou, H. Li, H. Xiao, L. Li, Q. Zhao, T. Yang, J. Zuo and W. Huang, Dalton Trans., 2013, 42, 5718.

7 (a) I. Stassen, N. Burtch, A. Talin, P. Falcaro, M. Allendorf and R. Ameloot, Chem. Soc. Rev., 2017, 46, 3185; (b) A. J. Howarth, A. W. Peters, N. A. Vermeulen, T. C. Wang, J. T. Hupp and O. K. Farha, Chem. Mater., 2017, 29, 26; (c) Y. J. Cui, B. Li, H. J. He, W. Zhou, B. L. Chen and G. D. Qian, Acc. Chem. Res., 2016, 49, 483.

8 (a) M. Eddaoudi, D. F. Sava, J. F. Eubank, K. Adil and V. Guillerm, Chem. Soc. Rev., 2015, 44, 228; (b) J. M. Zhou, W. Shi, N. Xu and P. Cheng, Inorg. Chem., 2013, 52, 8082; (c) J. M Zhou, W. Shi, H. M. Li, H. Li and P. Cheng, J. Phys. Chem. C, 2014, 118, 416; (d) J. Zhou, H. Li, H. Zhang, H. Li, W. Shi and P. Cheng, Adv. Mater., 2015, 27, 7072; (e) S. Y. Zhang, W. Shi, P. Cheng and M. J. Zaworotko, J. Am. Chem. Soc., 2015, 137, 12203; (f) H. Ma, L. Wang, J. Chen, X. Zhang, L. Wang, N. Xu, G. Yang and P. Cheng, Dalton Trans., 2017, 46, 3526; (g) L. Wang, G. Fan, X. Xu, D. Chen, L. Wang, W. Shi and P. Cheng, J. Mater. Chem. A, 2017, 5, 5541.

9 (a) J. S. Qin, S. J. Bao, P. Li, W. Xie, D. Y. Du, L. Zhao, Y. Q. Lan and Z. M. Su, Chem.-Asian J., 2014, 9, 749; (b) S. Dang, X. Min, W. T. Yang, F. Y. Yi, H. P. You and Z. M. Sun, Chem.-Eur. J., 2013, 19, 17172.

10 X. Zhou, H. Li, H. Xiao, L. Li, Q. Zhao, T. Yang, J. Zuo and W. Huang, Dalton Trans., 2013, 42, 5718.

11 X. M. Cao, N. Wei, L. Liu, L. Li and Z. B. Han, RSC Adv., 2016, 6, 19459.

12 X. L. Huang, L. Liu, M. L. Gao and Z. B. Han, RSC Adv., 2016, 6, 87945.

13 M. L. Gao, W. J. Wang, L. Liu, Z. B. Han, N. Wei, X. M. Cao and D. Q. Yuan, Inorg. Chem., 2017, 56, 511.

14 (a) S. Pramanik, C. Zheng, X. Zhang, T. J. Emge and J. Li, J. Am. Chem. Soc., 2011, 133, 4153; (b) B. Liu, W. P. Wu, L. Hou and Y. Y. Wang, Chem. Commun., 2014, 50, 8731; (c) F. Y. Yi, W. T. Yang and Z. M. Sun, J. Mater. Chem., 2012, 22, 23201. 
15 (a) X. D. Zhu, Y. Li, W. X. Zhou, R. M. Liu, Y. J. Ding, J. Lü and D. M. Proserpio, CrystEngComm, 2016, 18, 4530; (b) S. S. Nagarkar, B. Joarder, A. K. Chaudhari, S. Mukherjee and S. K. Ghosh, Angew. Chem., Int. Ed., 2013, 52, 2881.

16 (a) D. Tian, Y. Li, R. Y. Chen, Z. Chang, G. Y. Wang and X. H. Bu, J. Mater. Chem. A, 2014, 2, 1465; (b) J. D. Xiao, L. G. Qiu, F. Ke, Y. P. Yuan, G. S. Xu, Y. M. Wang and X. Jiang, J. Mater. Chem. A, 2013, 1, 8745; (c) K. Xiang,
Y. M. Li, C. H. Xu and S. H. Li, J. Mater. Chem. C, 2016, 4, 5578.

17 (a) S. M. Hu, H. L. Niu, L. G. Qiu, Y. P. Yuan, X. Jiang, A. J. Xie, Y. H. Shen and J. F. Zhu, Inorg. Chem. Commun., 2012, 17, 147; (b) S. S. Zhao, J. Yang, Y. Y. Liu and J. F. Ma, Inorg. Chem., 2016, 55, 2261; (c) H. Zhang, J. Yang, Y. Y. Liu, S. Y. Song and J. F. Ma, Cryst. Growth Des., 2016, 16, 3244. 